\title{
On the Origin of Species by Sympatric Speciation
}

Ulf Dieckmann (dieckman@iiasa.ac.at)

Michael Doebeli (doebeli@ubaclu.unibas.ch)

\section{Approved by}

Gordon J. MacDonald (macdon@iiasa.ac.at)

Director, IIASA

July 1999

Interim Reports on work of the International Institute for Applied Systems Analysis receive only limited review. Views or opinions expressed herein do not necessarily represent those of the Institute, its National Member Organizations, or other organizations supporting the work. 


\section{IIASA STUDIES IN ADAPTIVE DYNAMICS No. 35}



The Adaptive Dynamics Network at IIASA fosters the development of new mathematical and conceptual techniques for understanding the evolution of complex adaptive systems.

Focusing on these long-term implications of adaptive processes in systems of limited growth, the Adaptive Dynamics Network brings together scientists and institutions from around the world with IIASA acting as the central node.

Scientific progress within the network is reported in the IIASA Studies in Adaptive Dynamics series.

\section{THE ADAPTIVE DYNAMICS NETWORK}

The pivotal role of evolutionary theory in life sciences derives from its capability to provide causal explanations for phenomena that are highly improbable in the physicochemical sense. Yet, until recently, many facts in biology could not be accounted for in the light of evolution. Just as physicists for a long time ignored the presence of chaos, these phenomena were basically not perceived by biologists.

Two examples illustrate this assertion. Although Darwin's publication of "The Origin of Species" sparked off the whole evolutionary revolution, oddly enough, the population genetic framework underlying the modern synthesis holds no clues to speciation events. A second illustration is the more recently appreciated issue of jump increases in biological complexity that result from the aggregation of individuals into mutualistic wholes.

These and many more problems possess a common source: the interactions of individuals are bound to change the environments these individuals live in. By closing the feedback loop in the evolutionary explanation, a new mathematical theory of the evolution of complex adaptive systems arises. It is this general theoretical option that lies at the core of the emerging field of adaptive dynamics. In consequence a major promise of adaptive dynamics studies is to elucidate the long-term effects of the interactions between ecological and evolutionary processes.

A commitment to interfacing the theory with empirical applications is necessary both for validation and for management problems. For example, empirical evidence indicates that to control pests and diseases or to achieve sustainable harvesting of renewable resources evolutionary deliberation is already crucial on the time scale of two decades.

The Adaptive Dynamics Network has as its primary objective the development of mathematical tools for the analysis of adaptive systems inside and outside the biological realm. 


\section{IIASA STUDIES IN ADAPTIVE DYNAMICS}

No. 1 Metz JAJ, Geritz SAH, Meszéna G, Jacobs FJA, van Heerwaarden JS:

Adaptive Dynamics: A Geometrical Study of the Consequences of Nearly Faithful Reproduction.

IIASA Working Paper WP-95-099.

van Strien SJ, Verduyn Lunel SM (eds.): Stochastic and Spatial Structures of Dynamical Systems, Proceedings of the Royal Dutch Academy of Science (KNAW Verhandelingen), North Holland, Amsterdam, pp. 183-231 (1996).

No. 2 Dieckmann U, Law R:

The Dynamical Theory of Coevolution: A Derivation from Stochastic

Ecological Processes.

IIASA Working Paper WP-96-001.

Journal of Mathematical Biology (1996) 34, 579-612.

No. 3 Dieckmann U, Marrow P, Law R:

Evolutionary Cycling of Predator-Prey Interactions: Population Dynamics and the Red Queen.

Journal of Theoretical Biology (1995) 176, 91-102.

No. 4 Marrow P, Dieckmann U, Law R:

Evolutionary Dynamics of Predator-Prey Systems: An Ecological

Perspective.

IIASA Working Paper WP-96-002.

Journal of Mathematical Biology (1996) 34, 556-578.

No. 5 Law R, Marrow P, Dieckmann U:

On Evolution under Asymmetric Competition.

IIASA Working Paper WP-96-003.

Evolutionary Ecology (1997) 11, 485-501.

No. 6 Metz JAJ, Mylius SD, Diekmann O:

When Does Evolution Optimise? On the Relation between Types of Density Dependence and Evolutionarily Stable Life History Parameters.

IIASA Working Paper WP-96-004.

No. 7 Ferrière R, Gatto $\mathrm{M}$ :

Lyapunov Exponents and the Mathematics of Invasion in Oscillatory or Chaotic Populations.

Theoretical Population Biology (1995) 48, 126-171.

No. 8 Ferrière R, Fox GA:

Chaos and Evolution.

Trends in Ecology and Evolution (1995) 10, 480-485.

No. 9 Ferrière R, Michod RE:

The Evolution of Cooperation in Spatially Heterogeneous Populations.

IIASA Working Paper WP-96-029.

American Naturalist (1996) 147, 692-717. 
No. 10 Van Dooren TJM, Metz JAJ:

Delayed Maturation in Temporally Structured Populations with Non-Equilibrium Dynamics.

IIASA Working Paper WP-96-070.

Journal of Evolutionary Biology (1998) 11, 41-62.

No. 11 Geritz SAH, Metz JAJ, Kisdi E, Meszéna G:

The Dynamics of Adaptation and Evolutionary Branching.

IIASA Working Paper WP-96-077.

Physical Review Letters (1997) 78, 2024-2027.

No. 12 Geritz SAH, Kisdi E, Meszéna G, Metz JAJ:

Evolutionarily Singular Strategies and the Adaptive Growth and Branching of the Evolutionary Tree.

IIASA Working Paper WP-96-114.

Evolutionary Ecology (1998) 12, 35-57.

No. 13 Heino M, Metz JAJ, Kaitala V:

Evolution of Mixed Maturation Strategies in Semelparous Life-Histories: the Crucial Role of Dimensionality of Feedback Environment.

IIASA Working Paper WP-96-126.

Philosophical Transactions of the Royal Society of London Series B (1997) 352, 16471655.

No. 14 Dieckmann U:

Can Adaptive Dynamics Invade?

IIASA Working Paper WP-96-152.

Trends in Ecology and Evolution (1997) 12, 128-131.

No. 15 Meszéna G, Czibula I, Geritz SAH:

Adaptive Dynamics in a Two-Patch Environment: a Simple Model for Allopatric and Parapatric Speciation.

IIASA Interim Report IR-97-001.

Journal of Biological Systems (1997) 5, 265-284.

No. 16 Heino M, Metz JAJ, Kaitala V:

The Enigma of Frequency-Dependent Selection.

IIASA Interim Report IR-97-061.

Trends in Ecology and Evolution (1998) 13, 367-370.

No. 17 Heino M:

Management of Evolving Fish Stocks.

IIASA Interim Report IR-97-062.

Canadian Journal of Fisheries and Aquatic Sciences (1998) 55, 1971-1982.

No. 18 Heino M:

Evolution of Mixed Reproductive Strategies in Simple Life-History Models.

IIASA Interim Report IR-97-063.

No. 19 Geritz SAH, van der Meijden E, Metz JAJ:

Evolutionary Dynamics of Seed Size and Seedling Competitive Ability.

IIASA Interim Report IR-97-071. 
No. 20 Galis F, Metz JAJ:

Why are there so many Cichlid Species? On the Interplay of Speciation and Adaptive Radiation.

IIASA Interim Report IR-97-072.

Trends in Ecology and Evolution (1998) 13, 1-2.

No. 21 Boerlijst MC, Nowak MA, Sigmund K:

Equal Pay for all Prisoners. /The Logic of Contrition.

IIASA Interim Report IR-97-073.

AMS Monthly (1997) 104, 303-307. Journal of Theoretical Biology (1997) 185, 281-294.

No. 22 Law R, Dieckmann U:

Symbiosis without Mutualism and the Merger of Lineages in Evolution.

IIASA Interim Report IR-97-074.

Proceedings of the Royal Society of London Series B (1998) 265, 1245-1253.

No. 23 Klinkhamer PGL, de Jong TJ, Metz JAJ:

Sex and Size in Cosexual Plants.

IIASA Interim Report IR-97-078.

Trends in Ecology and Evolution (1997) 12, 260-265.

No. 24 Fontana W, Schuster P:

Shaping Space: The Possible and the Attainable in RNA Genotype-Phenotype Mapping.

IIASA Interim Report IR-98-004.

No. 25 Kisdi E, Geritz SAH:

Adaptive Dynamics in Allele Space: Evolution of Genetic Polymorphism by Small Mutations in a Heterogeneous Environment.

IIASA Interim Report IR-98-038.

No. 26 Fontana W, Schuster P:

Continuity in Evolution: On the Nature of Transitions.

IIASA Interim Report IR-98-039.

Science (1998) 280, 1451-1455.

No. 27 Nowak MA, Sigmund K:

Evolution of Indirect Reciprocity by Image Scoring. / The Dynamics of Indirect Reciprocity.

IIASA Interim Report IR-98-040.

Nature (1998) 393, 573-577.

No. 28 Kisdi E:

Evolutionary Branching Under Asymmetric Competition.

IIASA Interim Report IR-98-045.

No. 29 Berger U:

Best Response Adaptation for Role Games.

IIASA Interim Report IR-98-086.

No. 30 Van Dooren TJM:

The Evolutionary Ecology of Dominance-Recessivity

IIASA Interim Report IR-98-096. 
No. 31 Dieckmann U, O'Hara B, Weisser W:

The Evolutionary Ecology of Dispersal.

IIASA Interim Report IR-98-108.

Trends in Ecology and Evolution (1999) 14, 88-90.

No. 32 Sigmund K:

Complex Adaptive Systems and the Evolution of Reciprocation.

IIASA Interim Report IR-98-100.

No. 33 Posch M, Pichler A, Sigmund K:

The Efficiency of Adapting Aspiration Levels.

IIASA Interim Report IR-98-103.

No. 34 Mathias A, Kisdi É:

Evolutionary Branching and Coexistence of Germination Strategies.

IIASA Interim Report IR-99-014.

No. 35 Dieckmann U, Doebeli M:

On the Origin of Species by Sympatric Speciation

IIASA Interim Report IR-99-013.

Nature (1999) 400, 354-357.

Issues of the IIASA Studies in Adaptive Dynamics series can be obtained free of charge.

Please contact:

Adaptive Dynamics Network

International Institute for Applied Systems Analysis

Schlossplatz 1

A-2361 Laxenburg

Austria

Telephone +432236 807, Telefax +432236 71313, E-Mail adn@iiasa.ac.at, Internet http://www.iiasa.ac.at/Research/ADN 


\begin{abstract}
Understanding speciation is a fundamental biological problem. It is believed that many species originated through allopatric divergence in geographically isolated populations of the same ancestral species ${ }^{1-3}$. In contrast, the possibility of sympatric speciation has often been dismissed, partly because of theoretical difficulties ${ }^{2,3}$. Most previous models analysing sympatric speciation concentrated on particular aspects of the problem while neglecting others ${ }^{4-10}$. We present a model which integrates a novel combination of different features and shows that sympatric speciation is a likely outcome of competition for resources. We use explicit multilocus genetics to describe sexual reproduction in an individual-based model, and we consider the evolution of assortative mating depending either on the ecological character affecting intraspecific resource competition or on a selectively neutral marker trait. In both cases, evolution of assortative mating often leads to reproductive isolation between ecologically diverging subpopulations. When assortative mating depends on a marker trait, and is therefore not directly linked to resource competition, speciation occurs when genetic drift breaks the linkage equilibrium between marker and ecological trait. Our theory conforms well with mounting empirical evidence for the sympatric origin of many species ${ }^{10-18}$.
\end{abstract}




\title{
About the Author
}

\author{
Ulf Dieckmann \\ Adaptive Dynamics Network \\ A-2361 Laxenburg, Austria \\ Michael Doebeli \\ Zoology Institute \\ University of Basel \\ Rheinsprung 9 \\ CH-4051 Basel, Switzerland
}

International Institute for Applied Systems Analysis

\section{Acknowledgment}

We thank Hans Metz, Richard Law, Mikko Heino, Steve Stearns, John Maynard Smith, Nancy Knowlton and three anonymous referees for helpful comments, and Uli Schliewen, Konny Rasmussen, and Diethard Tautz for discussions. The order of authors is alphabetical. 


\title{
On the Origin of Species by Sympatric Speciation
}

\author{
Ulf Dieckmann \\ Michael Doebeli
}

The theory of adaptive dynamics ${ }^{19-22}$ is a general framework for studying phenotypic evolution driven by ecological interactions. One of the phenomena unraveled by adaptive dynamics is evolutionary branching, during which directional selection drives a monomorphic population to a phenotype where ecological interactions induce disruptive selection and a subsequent split into two coexisting phenotypic clusters (Fig. 1a). Evolutionary branching explains the dynamic emergence and perpetuity of disruptive selection and serves as a unifying concept for understanding the evolution of polymorphisms. It is found in a wide range of models for asexual populations (see ref. 22 and 23 for examples). Here we demonstrate that evolutionary branching also occurs in sexual populations and thus leads to a general theory for sympatric speciation.

We start from assumptions likely to be satisfied in many natural populations. Individuals vary in a quantitative character $x$ determining resource use, as e.g. when beak size in birds determines the size of seeds consumed. Populations consisting of individuals of a given trait value $x$ have density-dependent logistic growth with carrying capacity $K(x)$. We assume that the resource distribution $K(x)$ is unimodal and varies according to a Gaussian function $N\left(x_{0}, \sigma_{K}\right)$ with maximum at an intermediate phenotype $x_{0}$ and variance $\sigma_{K}^{2}$. In polymorphic populations consisting of individuals with different trait values, dissimilar individuals interact only weakly, as e.g. when birds with different beak sizes eat different types of seeds. That is, competition is not only density- but also frequency-dependent, and rare phenotypes experience less competition than common phenotypes. Specifically, we assume that the strength of competition between individuals declines with phenotypic distance according to a Gaussian function $N\left(0, \sigma_{C}\right)$ with maximum at 0 and variance $\sigma_{C}^{2}$.

These assumptions are integrated into an asexual individual-based model in which each individual is characterized by its trait value $x$. Individuals give birth at a constant rate and die at a rate that is determined by frequency- and densitydependent competition (see Methods). Evolutionary dynamics occur because offspring phenotypes may deviate slightly from parent phenotypes. The quantitative character first evolves to the value $x_{0}$ with maximal carrying capacity. After that, two things can happen: either $x_{0}$ is evolutionarily stable and evolution comes to a halt at $x_{0}$, or $x_{0}$ is actually a fitness minimum and can be invaded by all nearby phenotypes $^{19,21,22}$. In the latter case, evolutionary branching occurs (Fig. 1a). This happens for $\sigma_{C}<\sigma_{K}$, i.e., if the curvature of the carrying capacity at its maximum is less than that of the competition function. Then the advantage of deviating from the crowded optimal phenotype $x_{0}$ more than compensates for the disadvantage of a lower carrying capacity. 

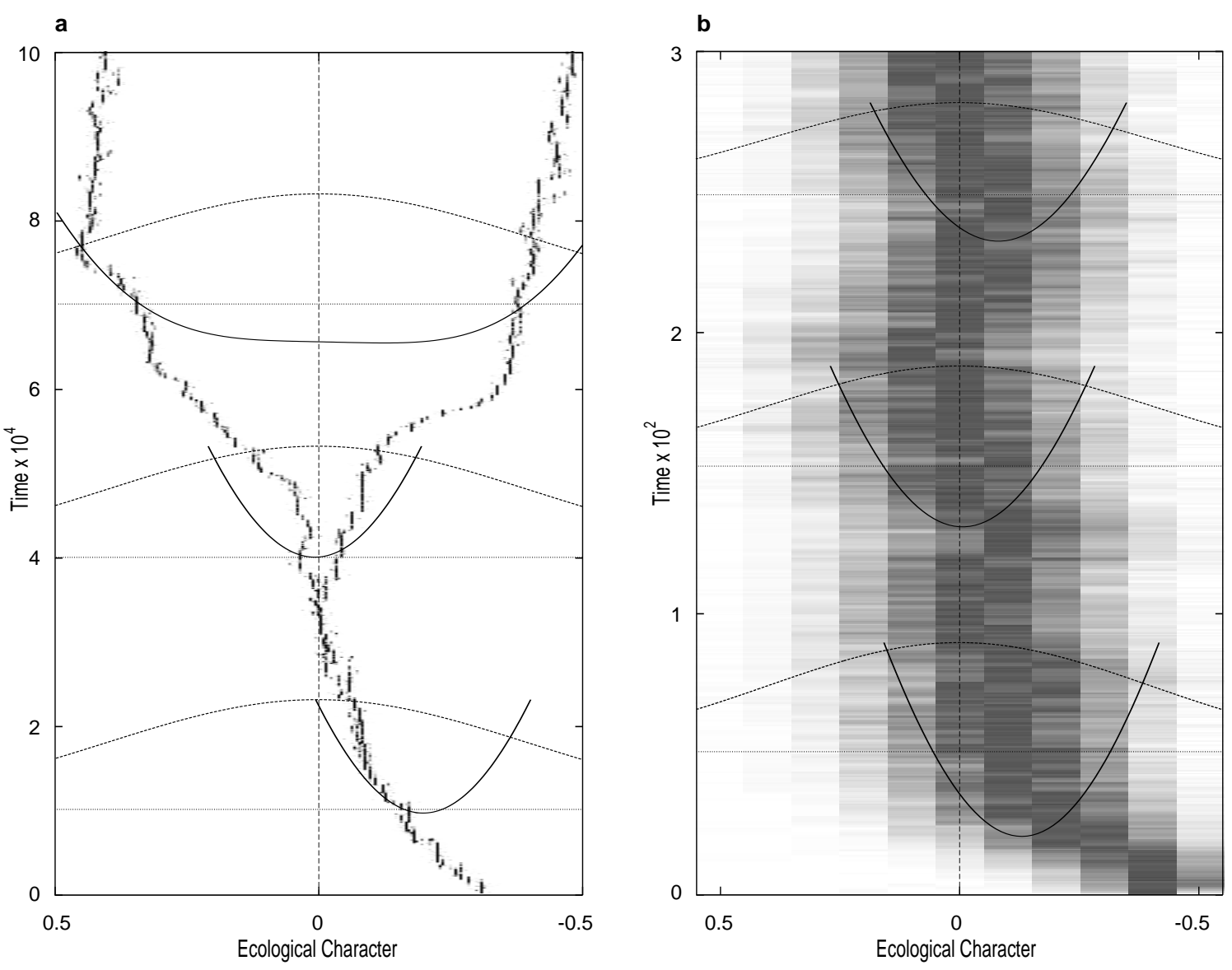

Figure 1: a) Evolutionary branching in the individual-based asexual model: at the branching point $x_{0}=0$, the population splits into two morphs. Three insets show fitness functions (continuous curves) generated by the ecological interactions at different points in time (indicated by horizontal dotted lines). Selection changes from directional to disruptive when evolution reaches $x_{0}$. The resource distribution $K(x)$ has its maximum at $x_{0}$ and is shown for comparison (dashed curve).

b) Same as a) but with multilocus genetics for the ecological character and random mating. Shading represents phenotype distributions (5 diploid and diallelic loci result in 11 possible phenotypes). Despite disruptive selection at the branching point (see insets), branching does not occur. 
Sexual reproduction is incorporated by assuming that character values are determined by many additive, diploid loci with two alleles, + and - , and are proportional to the number of + alleles. Offspring inherit maternal and paternal alleles at each locus independently (free recombination). As in the asexual case, the sexual population evolves to a mean phenotype $x_{0}$. If mating is random, however, evolutionary branching does not occur for any values of $\sigma_{K}$ and $\sigma_{C}$ : the split into two distinct phenotypic morphs is prevented by the continual generation of intermediate phenotypes through recombination (Fig. 1b). Thus, in sexual populations non-random mating is a prerequisite for evolutionary branching ${ }^{24}$.

To model the evolution of assortative mating we assume that individuals express an additional quantitative character which determines mating probabilities according to two scenarios. In the first, mating probabilities are based on similarity in the ecological character, and in the second they are based on similarity in a third, ecologically neutral 'marker' trait (see Methods). Mating character and marker trait are also determined by many additive diallelic loci. Individuals having an intermediate mating character mate randomly. Individuals carrying mostly - alleles at the mating loci mate disassortatively, hence are more likely to mate with individuals with very different ecological or marker phenotypes, while individuals carrying mostly + alleles at the mating loci mate assortatively: the probability of mating increases with phenotypic similarity to the partner (Fig. 2).

Fig. 3a shows the evolutionary dynamics of an initially randomly mating population when mating probabilities depend on the ecological character. While this character evolves to $x_{0}$, the mating character initially changes only slowly but picks up speed and evolves towards positive assortativeness when the ecological character reaches $x_{0}$. Once assortativeness is strong enough, the population splits into two ecologically different morphs which eventually are almost completely reproductively isolated. These results confirm and extend those of ref. 24 and occur because near the dynamically emerging fitness minimum at $x_{0}$, selection favors mechanisms that allow for a split in the phenotype distribution and hence for a departure from the fitness minimum. Assortative mating is such a mechanism, because it prevents the generation of intermediate offspring phenotypes from extreme parent phenotypes. Parameter requirements for evolutionary branching in sexual populations appear to be only slightly more restrictive than in the asexual case (Fig. 4).

When assortative mating depends on the ecological character speciation is not hindered by recombination between mating loci and ecological loci. However, when mating depends on an ecologically neutral marker trait, a linkage disequilibrium between marker loci and ecological loci, leading to a correlation between marker trait and ecological character, is required for the evolution of assortative mating and for speciation. Classical, deterministic models (e.g., Felsenstein's 'two-allele' models) predict that such linkage disequilibria are unlikely because of recombination between ecological and marker loci ${ }^{3,6}$. In our individual-based model, however, genetic drift due to stochastic demographic effects readily leads to speciation despite the opposing force of recombination. Fig. 3b shows the adaptive dynamics when mating probabilities depend on a neutral marker trait. Genetic drift temporarily results in small and localized linkage disequilibria between some marker loci and some ecological loci. Positive and negative correlations both select for assortative mating, 


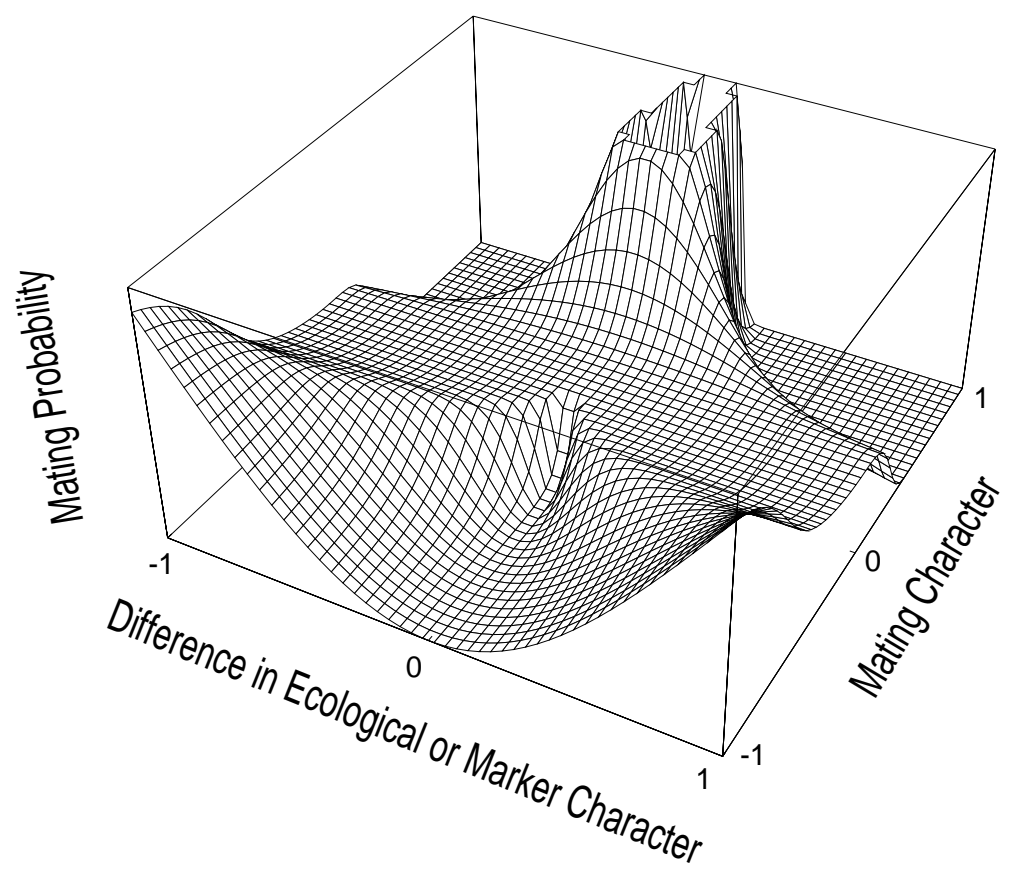

Figure 2: Mating probabilities as determined by mating character and difference in ecological or marker character between mates. The mating character $m$ is scaled to vary between -1 (all - alleles) and +1 (all + alleles). Mating probabilities vary with differences in either ecological or marker character, depending on the scenario. If the mating character in the focal individual is close to +1 , it has a high probability of mating with similar individuals. If its mating character is close to -1 , it is more likely to mate with dissimilar individuals. Intermediate mating characters (close to 0 ) correspond to random mating. 

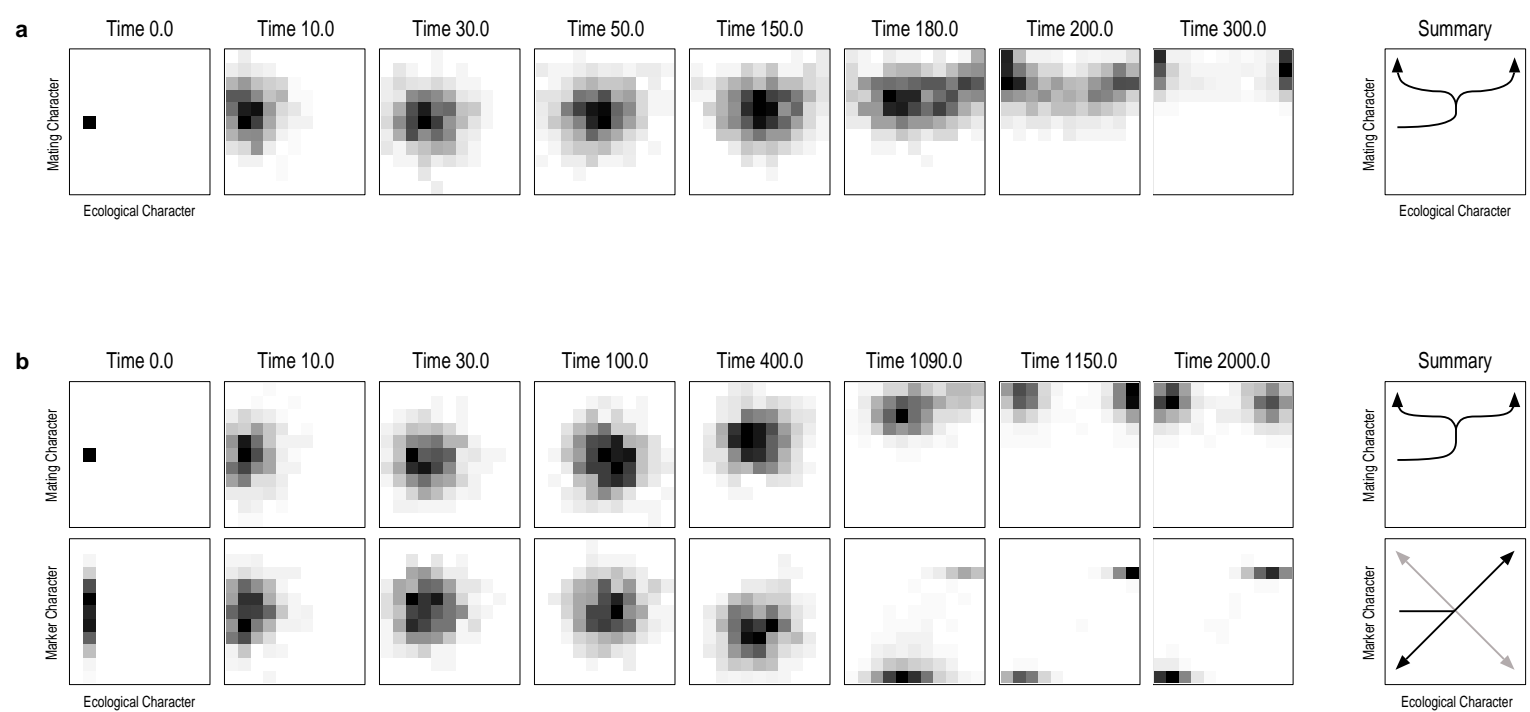

Figure 3: Evolutionary branching in sexual populations

a) First scenario: mating probabilities (vertical axes) depend on the ecological character (horizontal axes), which first evolves to intermediate values (50 generations). Then the mean mating character increases to positive values (180 generations) and induces a bimodal split in the ecological character (200 generations).

b) Second scenario: mating probabilities (vertical axes in upper panels) depends on a marker trait (vertical axes in lower panels). The ecological trait (horizontal axes in all panels) first evolves to intermediate values (100 generations). Due to temporary correlations between marker and ecological trait, assortative mating increases, which in turn magnifies these correlations (generations 400-1090). This positive feedback eventually leads to speciation (1150 generations).

In the second scenario, branching typically takes longer than in the first. The summary panels depict the evolution of mean character values schematically. Gray arrows in the bottom summary panel show an alternative, equally likely, evolution of linkage disequilibrium between ecological and marker character. 


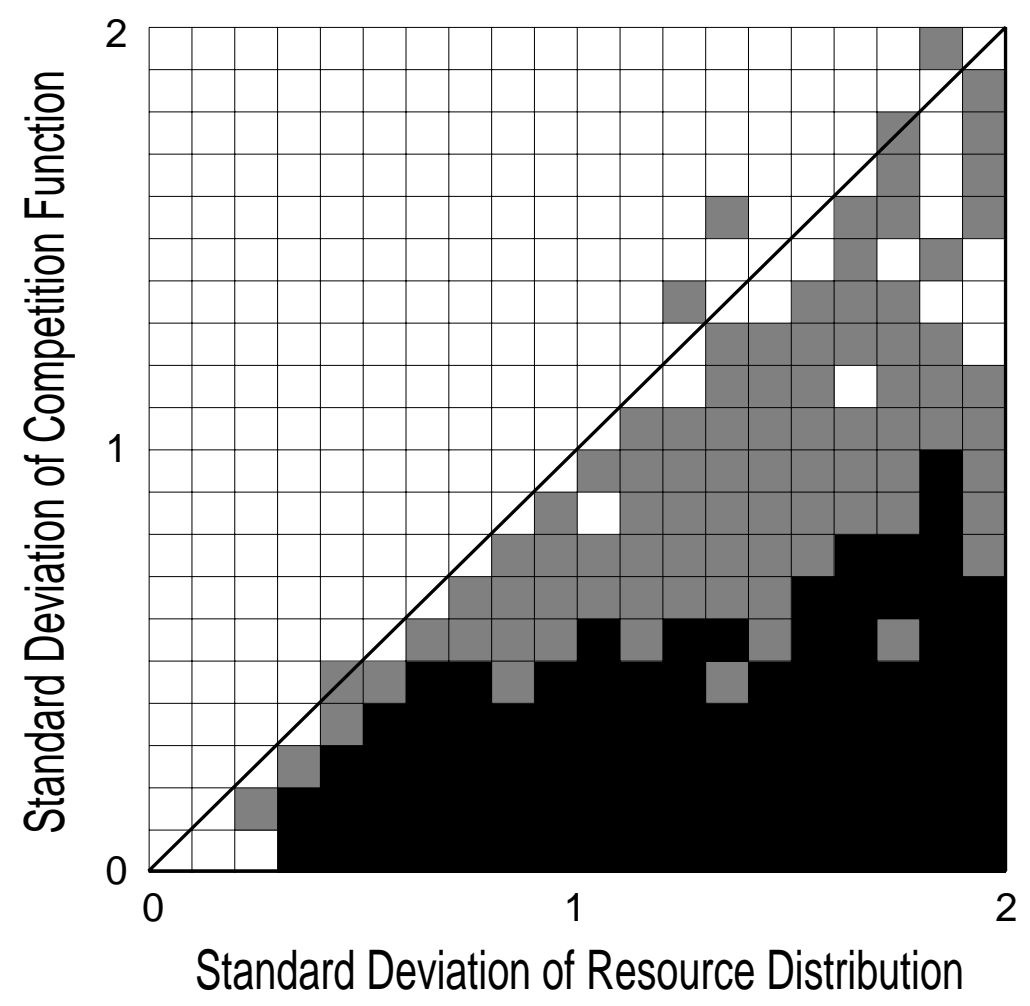

Figure 4: Combinations of standard deviations $\sigma_{K}$ and $\sigma_{C}$ of resource distribution $K(x)$ and competition function $C(x)$, respectively, that allow for evolutionary branching. Analytical results are available for the asexual model (see Methods) and predict branching for $\sigma_{C}<\sigma_{K}$, i.e. below the diagonal (black triangle). Conditions for branching in sexual populations (within 20,000 generations) are shown in gray when mating probabilities depend on the ecological character and in black when they depend on a marker trait. 

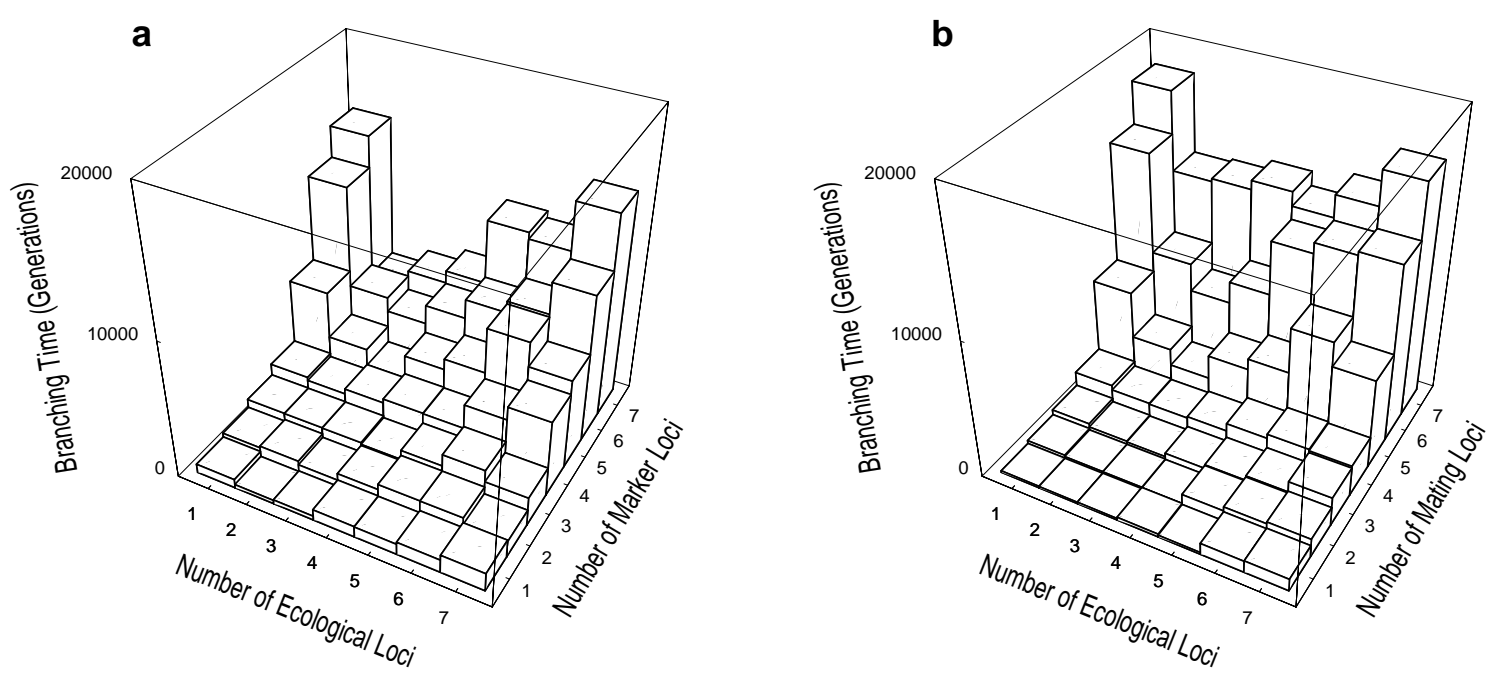

Figure 5: Average waiting times for evolutionary branching with different numbers of loci when assortative mating depends on a marker trait.

a) Variable numbers of loci for marker and ecological trait with a fixed number of loci (5) for assortative mating;

b) Variable numbers of loci for assortative mating and ecological trait with a fixed number of loci (5) for the marker trait.

Other parameters are as in Fig. 3; each column represents the average waiting time from 60 simulation runs.

which in turn magnifies the local disequilibria into a global linkage disequilibrium between marker and ecological trait. This feedback eventually induces the sympatric split into reproductively isolated phenotypic clusters. Thus, stochastic fluctuations in finite populations can spontaneously break the symmetry of linkage equilibria observed in deterministic models. Recombination between marker loci and ecological loci implies that parameter requirements for evolutionary branching are more restrictive when mate choice is based on a neutral marker than when it is based on the ecological trait (Fig. 4).

The effects of stochasticity on evolutionary branching are further illustrated by varying the number of loci determining the quantitative characters (Fig. 5). Evolutionary branching is more likely when there are fewer loci, for then the phenotypic effects of genetic drift are larger (an exception occurs with only one ecological locus: with only three phenotypes, sufficiently strong fluctuations arise more rarely). Branching triggered by drift becomes less likely in very large populations where stochastic effects become small.

Our results extend and contrast previous insights ${ }^{6,8,9,24-26}$ by showing that competition for unimodal resources can initiate sympatric speciation even if assortative mating depends on an ecologically neutral marker trait. The results are robust against changes in the models such as varying numbers of loci (Fig. 5), assuming different mutation rates per locus, assuming different relations between the number of + alleles on the mate choice loci and the degree of assortativeness (see Methods), and assuming different functions for the carrying capacities, $K(x)$, and for the 
strength of competition, $C(x)$, while maintaining their qualitative characteristics. Evidence is accumulating that ecology is important for speciation ${ }^{18,27,28}$, and our theory may provide an integrative framework for understanding otherwise puzzling evidence for monophyletic origins of many sympatric species including cichlids ${ }^{11,12}$, sticklebacks ${ }^{13,16,27}$, snails ${ }^{14}$, giant senecios ${ }^{15}$, and anolis lizards ${ }^{17}$. In all these cases it is likely that frequency-dependent mechanisms are important determinants of the species' ecologies. Therefore, assortative mating based on ecologically important traits such as body size (e.g. in sticklebacks ${ }^{29}$ ) or on marker traits that covary with ecological traits (e.g. coloration or breeding behaviour in cichlids ${ }^{30}$ ) could have led to the formation of new species in accordance with the theory presented here. We expect our theory to work best in relatively recently colonized habitats, in which sympatric divergence is not strongly opposed by competition from other species already present. In fact, a striking example of incipient sympatric speciation due to ecological interactions in a new habitat has recently been documented in a pair of cichlid morphs (Schliewen et al., submitted), in which restricted gene flow has evolved through size-assortative mating. The mechanisms of speciation are rarely as clear as in this example, but our theoretical evidence generally suggests a prominent role for ecologically driven speciation in sympatry.

\section{Methods}

Deterministic dynamics of a resident population of phenotype $x$ are

$$
\frac{d N(x, t)}{d t}=r \cdot N(x, t) \cdot\left[1-\frac{N(x, t)}{K(x)}\right]
$$

where $N(x, t)$ is population size at time $t$. The carrying capacity, $K(x)=K_{0}$. $\exp \left(-\frac{\left(x-x_{0}\right)^{2}}{2 \sigma_{K}^{2}}\right)$, is the stable equilibrium. When a rare mutant $y$ appears in a resident $x$ at carrying capacity $K(x)$, it competes with the discounted density $C(x-y)$. $K(x)$, where $C(x-y)=\exp \left(-\frac{(x-y)^{2}}{2 \sigma_{C}^{2}}\right)$ describes the strength of competition between phenotypes. Therefore, the per capita growth rate $s(y, x)$ of the rare mutant $y$ is $r \cdot\left[1-\frac{C(x-y) \cdot K(x)}{K(y)}\right]$. The derivative $\left.\frac{\partial s(y, x)}{\partial y}\right|_{y=x}=r \cdot \frac{K^{\prime}(x)}{K(x)}$ of $s(y, x)$ with respect to the mutant $y$ and evaluated at the resident $x$ is positive for $x<x_{0}$ and negative for $x>x_{0}$. Therefore, $x_{0}$ is an attractor for the adaptive dynamics ${ }^{19,21,22}$. In addition, if $s\left(y, x_{0}\right)$ has a minimum at $y=x_{0}$, then $x_{0}$ is a branching point ${ }^{19,21,22}$. This happens if and only if $\sigma_{C}<\sigma_{K}$.

These analytical predictions are confirmed by the individual-based asexual model, in which individuals are assigned a phenotype $x$, give birth at a rate $r$, and die at a rate $\frac{r}{K(x)} \cdot \sum_{y} N(y, t) \cdot C(x-y)$, where the sum weighs all individuals by their competitive impact on $x$. Offspring have the same phenotype as their parent, except when a mutation occurs (at rate 0.001), in which case their phenotype is chosen from a normal distribution $N\left(x, \frac{1}{20}\right)$, where $x$ is the parent phenotype.

In sexual populations, birth and death rates are calculated similarly. Individuals are assigned up to three diploid genotypes with 5 diallelic loci each (variation in loci number is analyzed in Fig. 5). The first set of loci determines the ecological character $x$, the second set determines mating probabilities, and the third encodes the marker trait. The mating character $m$ is given by the difference between the 
number of + and - alleles divided by the total number of alleles. If assortative mating depends on the ecological trait, then, for $m>0$, mating probabilities fall off with a difference in the ecological trait according to a Gaussian function $N\left(x, \sigma_{a}\right)$ with mean equal to the focal individual's ecological trait and variance $\sigma_{a}=\frac{1}{20 \mathrm{~m}^{2}}$. If $m=0$, the focal individual mates randomly. If $m<0$, then mating probabilities increase with ecological difference according to the function $1-N\left(x, \sigma_{d}\right)$, where $\sigma_{d}=\frac{1}{m^{2}}$ (Fig. 2). If assortative mating depends on the marker trait, then the third set of loci replaces the ecological trait in determining mating probabilities, which then depend on similarity in the marker trait. In order to avoid a bias against marginal phenotypes in the population, mating probabilities are normalized, so that the sum of mating probabilities over all potential partners is 1 for all phenotypes. A 50:50 sex ratio is assumed at all times. At each locus, one offspring allele is chosen randomly from the two maternal alleles and the other from the two paternal alleles at this locus. With a small probability (0.001), a mutation occurs in the inherited alleles and reverses their value. Other parameter values used for the figures are $r=1, K_{0}=500, \sigma_{K}=1$, and $\sigma_{C}=0.4$ (variation in the latter two parameters is analyzed in Fig. 4).

\section{References}

1. Mayr, E. Animal species and evolution. (Harvard University Press, Cambridge, Mass., 1963).

2. Coyne, J.A. Genetics and speciation. Nature 355, 511-515 (1992).

3. Rice, W.R. \& Hostert, E.E. Laboratory experiments on speciation - what have we learned in 40 years. Evolution 47, 1637-1653 (1993).

4. Maynard Smith, J. Sympatric speciation. Am. Nat. 100, 637-650 (1966).

5. Rosenzweig, M.L. Competitive speciation. Biol. J. Linn. Soc. (Lond.) 10, 275-289 (1978).

6. Felsenstein, J. Skepticism towards Santa Rosalia, or why are there so few kinds of animals? Evolution 35, 124-138 (1981).

7. Seger, J. Intraspecific resource competition as a cause of sympatric speciation. In: Evolution. Essays in honour of John Maynard Smith. (Greenwood, P.J., Harvey, P.H. \& Slatkin, M. eds., Cambridge University Press, Cambridge, 1985).

8. Kondrashov, A.S. Multilocus model of sympatric speciation III. Computer simulations. Theor. Pop. Biol. 29, 1-15 (1986).

9. Johnson, P.A., Hoppenstaedt, F.C., Smith, J.J. \& Bush, G.L. Conditions for sympatric speciation: a diploid model incorporating habitat fidelity and nonhabitat assortative mating. Evol. Ecol. 10, 187-205 (1996).

10. Bush, G.L. Sympatric speciation in animals - new wine in old bottles. Trends Ecol. Evol. 9, 285-288 (1994). 
11. Meyer, A., Kocher, T.D., Basasibwaki, P. \& Wilson, A.C. Monophyletic origin of Lake Victoria cichlid fishes suggested by mitochondrial DNA sequences. Nature 347, 550-553 (1990).

12. Schliewen, U.K., Tautz, D. \& Pääbo, S. Sympatric speciation suggested by monophyly of crater lake cichlids. Nature 368, 629-623 (1994).

13. Schluter, D. Experimental evidence that competition promotes divergence in adaptive radiation. Science 266, 798-801 (1994).

14. Johannesson, K., Rolan-Alvarez, E., Ekendahl, A. Incipient reproductive isolation between two sympatric morphs of the intertidal snail Littorina saxatilis. Evolution 49, 1180-1190 (1995).

15. Knox, E.B. \& Palmer, J.D. Chloroplast DNA variation and the recent radiation of giant senecios (Asteraceae) on the tall mountains of Eastern Africa. Proc. Natl. Acad. Sci. USA 92, 10349-10353 (1995).

16. Taylor, E.B. \& McPhail, J.D. Evolutionary history of an adaptive radiation in species pairs of threespine sticklebacks (Gasterosteus): insights from mitochondrial DNA. Biol. J. Linn. Soc. 66, 271-291 (1999).

17. Losos, J.B., Jackman, T.R., Larson, A., de Queiroz, K. \& Rodríguez-Schettino, L. Contingency and determinism in replicated adaptive radiations of island lizards. Science 279, 2115-2118 (1998).

18. Orr, M.R. \& Smith, T.B. Ecology and speciation. Trends Ecol. Evol. 13, 502-506 (1998).

19. Metz J.A.J., Geritz, S.A.H., Meszéna, G., Jacobs, F.J.A. \& van Heerwaarden, J.S. Adaptive dynamics: a geometrical study of the consequences of nearly faithful reproduction. pp. 183-231 in Stochastic and Spatial Structures of Dynamical Systems. (S.J. van Strien and S.M. Verduyn Lunel eds., North Holland, Amsterdam, 1996).

20. Dieckmann, U. \& Law, R. The dynamical theory of coevolution: a derivation from stochastic ecological processes. J. Math. Biol. 34, 579-612 (1996).

21. Dieckmann, U. Can adaptive dynamics invade? Trends Ecol. Evol. 12, 128131 (1997).

22. Geritz, S.A.H., Kisdi, E., Meszéna, G. \& Metz, J.A.J. Evolutionarily singular strategies and the adaptive growth and branching of the evolutionary tree. Evol. Ecol. 12, 35-57 (1998).

23. Doebeli, M. \& Ruxton, G.D. Evolution of dispersal rates in metapopulation models: branching and cyclic dynamics in phenotype space. Evolution 51, 1730-1741 (1997).

24. Doebeli, M. A quantitative genetic competition model for sympatric speciation. J. Evol. Biol. 9, 893-909 (1996). 
25. Turner, G.F. \& Burrows, M.T. A model of sympatric speciation by sexual selection. Proc. Roy. Soc. Lond. B 260, 287-292 (1995).

26. Kondrashov, A.S. \& Shpak, M. On the origin of species by means of assortative mating. Proc. Roy. Soc. Lond. B 265, 2273-2278 (1998).

27. Schluter, D. \& McPhail, J.D. Character displacement and replicate adaptive radiation. Trends Ecol. Evol. 8, 197-200 (1993).

28. Schluter, D. \& Nagel, L.M. Parallel speciation by natural selection. Am. Nat. 146, 292-301 (1995).

29. Nagel, L.M. \& Schluter, D. Body size, natural selection, and speciation in sticklebacks. Evolution 52, 209-218 (1998).

30. Seehausen, O., van Alphen, J.J.M. \& Witte, F. Cichlid fish diversity threatened by eutrophication that curbs sexual selection. Science 277, 1808-1811 (1997). 